\title{
Impact of CYP2D6 polymorphisms in tamoxifen adjuvant breast cancer treatment
}

\author{
T. Ramón y Cajal $\cdot$ A. Altés $\cdot$ L. Paré $\cdot$ E. del Rio $\cdot$ \\ C. Alonso $\cdot$ A. Barnadas $\cdot$ M. Baiget
}

Received: 30 December 2008/ Accepted: 20 January 2009/Published online: 3 February 2009

(C) Springer Science+Business Media, LLC. 2009

\begin{abstract}
The aim of this study is to evaluate the impact of CYP2D6 genotyping in predicting disease-free survival and toxicity in breast cancer patients treated with adjuvant tamoxifen. DNA from 91 patients was genotyped using the AmpliChip CYP450 GeneChip ${ }^{\circledR}$, Roche that facilitates the classification of individuals by testing 27 alleles. When patients were grouped into group $1(* 4 / * 4, * 4 / * 41, * 1 / * 5$ and $* 2 / * 5$ ) and group 2 (the remaining genotypes), a significant difference in disease-free survival (DFS) was observed between groups $(P=0.016)$. The mean DFS in group 1 was 95 months in contrast with 119 months in group 2. No significant relationship was found between the CYP2D6 genotype classification and severe, mild or no toxicity $(P=0.2)$. Nevertheless, severe, and mild toxicity was more frequent among poor metabolizer patients than in patients with a normal metabolizer pattern (18.8 and 43.8\% vs. 10.7 and $36 \%$, respectively). In breast cancer, patients treated with adjuvant tamoxifen, non-functional and severely impaired CYP2D6 variants are associated with a worse DFS and with a higher frequency of severe and mild toxicities. Larger studies of the CYP2D6 genotype-clinical
\end{abstract}

T. Ramón y Cajal $\cdot$ C. Alonso · A. Barnadas

Servicio de Oncología Médica, Hospital de la Santa Creu i Sant

Pau, Universitat Autónoma de Barcelona, Barcelona, Spain

A. Altés

Servicio de Hematología, Fundació Althaia, Manresa,

Barcelona, Spain

L. Paré · E. del Rio · M. Baiget $(\bowtie)$

Servicio de Genética, Hospital de la Santa Creu i Sant Pau,

Universitat Autónoma de Barcelona, Barcelona, Spain

e-mail: mbaiget@santpau.es; mbaiget@santpau.cat outcomes association are needed to complement initial results.

Keywords Breast cancer - Tamoxifen · CYP2D6 genotype $\cdot$ Pharmacogenetics

\section{Introduction}

The anti-estrogen tamoxifen (TMX) is a fundamental piece of standard adjuvant and palliative systemic therapy for patients with steroid hormone receptor-positive breast tumors [1]. Adjuvant tamoxifen significantly decreases relapse rates and mortality in pre-and postmenopausal patients [2]. For many years, it was widely believed that the clinical activity of TMX was largely mediated by 4-hydroxy-TMX, the metabolite that results from the action of different P450 cytochromes on the TMX molecule. Nevertheless, Stearns et al. [3] identified an active metabolite of TMX, 4-hydroxy- $N$-desmethyl-tamoxifen (endoxifen) that is present in higher concentrations than 4-hydroxyTMX in the blood of women on chronic adjuvant TMX therapy. They demonstrated that the primary route of metabolism from TMX to endoxifen is $N$-demethylation by cytochrome P4503A to $N$-desmethyl-tamoxifen, followed by hydroxylation by cytochrome P4502D6. They also showed that endoxifen suppressed estradiol-stimulated MCF7 proliferation, with potency comparable to that of 4-hydroxy-tamoxifen. Because baseline steady-state levels of endoxifen were present at much higher concentrations than those of 4-hydroxy-tamoxifen, endoxifen may be even more important than 4-hydroxy-TMX in mediating the anticancer effects of tamoxifen. These authors also demonstrated that coadministration of paroxetin decreased the 
Table 1 Frequencies of the most prevalent CYP2D6 alleles different ethnic groups

Data from Ref. [4-7]

\begin{tabular}{lllll}
\hline CYP2D6 allele & Enzymatic activity & Caucasian $(\%)$ & African-American (\%) & Asian (\%) \\
\hline$* 1$ & Functional & $30-40$ & $28-50$ & $20-40$ \\
$* 2$ & Functional & $20-35$ & $10-80$ & $9-20$ \\
$* 35$ & Functional & $4-6$ & - & - \\
$* 3$ & Nonfunctional & $1-4$ & $0-0,5$ & $0.8-1$ \\
$* 4$ & Nonfunctional & $12-23$ & $2-7$ & $0.5-3$ \\
$* 5$ & Nonfunctional & $1.5-7$ & $0.5-6$ & $4-6$ \\
$* 6$ & Nonfunctional & $0.5-1$ & 0 & - \\
$* 4 \times \mathrm{n}$ & Nonfunctional & $0.1-0.5$ & 0.9 & - \\
$* 9$ & Reduced & $0-3$ & 0 & 3 \\
$* 10$ & Reduced & $2-8$ & $3-8$ & $40-70$ \\
$* 17$ & Reduced & $0.1-0.3$ & $10-30$ & 0.5 \\
$* 41$ & Reduced & 8 & - & - \\
$* 1 \times \mathrm{n}$ & Increased & $0.2-1$ & $2-5$ & 0.5 \\
$* 2 \times \mathrm{n}$ & Increased & $0.5-1.5$ & $1.5-2.5$ & $0-1$ \\
\hline
\end{tabular}

plasma concentration of endoxifen and they suggest that CYP2D6 genotype and drug interactions should be considered in women treated with TMX.

The CYP2D6 gene is highly polymorphic and over 70 major alleles are known to date. Many of these are associated with increased, decreased, or abolished function of the final gene product. The CYP2D6 phenotypes associated with these different alleles include poor (PM), intermediate (IM), extensive (EM) and ultrarapid (UM) metabolizers. Some of the most common and important variant alleles distributed in different ethnic groups are listed in Table 1 [4-7] and these variant alleles are presented on the homepage of the human CYP allele nomenclature committee [8] Carriers of any two of approximately 20 known null alleles are phenotypic poor metabolizers, representing $7-10 \%$ of the European and North American Caucasian population. One of the most important functionally altered null variants is CYP2D6*4 (12-21\% in Caucasians). Alleles associated with reduced enzyme activity include CYP 2D6*10 (up to $70 \%$ in Asians) and CYP2D6*17 (10-30\% in African and African Americans). Individuals at the high end of the activity spectrum (UM) carry gene duplications and multiduplications of functional alleles leading to higher CYP2D6 expression and enzyme activity. These alleles have a relatively low frequency in Caucasians and Asians, but are commonly observed in Ethiopians.

In 2004, the FDA approved the AmpliChip CYP450 GeneChip ${ }^{\circledR}$, Roche as a biotechnology tool that facilitates the classification of individuals into CYP2D6 phenotypes by testing 27 alleles. The aim of this study was to evaluate the impact of the CYP2D6 genotyping using this microarray in predicting event-free survival and toxicity in breast cancer patients treated with adjuvant tamoxifen.

\section{Patients and methods}

Patients

In this retrospective study, performed in a single center, we selected patients diagnosed with primary invasive breast cancer between 1996 and 1998. Those included in the study were the 91 patients that came to our hospital during 2007 for outpatient follow up. Samples were collected on this occasion. Two treatment groups were defined: an adjuvant monotherapy-tamoxifen group and an adjuvant tamoxifen and concomitant chemotherapy group. Additionally, patients from both groups were treated with radiotherapy. Because information on comedication of patients receiving SSRIs (selective serotonin reuptake inhibitors) was incomplete, it was not included in the analyses. Written informed consent was obtained from all patients, and the study was approved by the Institutional Ethics Committee.

\section{Clinical parameters}

Relevant clinical data (age, menopausal status, histological type, and grade, tumour size, lymph node involvement, hormone receptor status) were obtained from clinical records. Disease free survival (DFS) was calculated from the beginning of therapy to: (a) the time of relapse, (b) the appearance of a contralateral breast cancer or (c) death.

Toxicity data (cebrovascular accident, pulmonary embolism, deep vein thrombosis, endometrial carcinoma, toxicodermia, Intrauterine polyp, transaminitis, amenorrhea, ovaric cists, steatosis and vaginitis) were also collected from clinical records. To evaluate the adverse effects associated with tamoxifen administration, we defined three groups of patients: those with no toxicity; those with severe toxicity 
(cerebrovascular accident, pulmonary embolism, deep vein thrombosis, endometrial carcinoma, toxicodermia) and those with the remaining adverse effects caused by the drug, (Table 2).

\section{Genotyping}

After receiving informed consent, we obtained EDTAwhole blood from 91 patients and DNA was isolated by the salting out procedure [9]. We used a microarray hybridization method (AmpliChip CYP450 GeneChip ${ }^{\circledR}$, Roche) for the detection of different polymorphisms in the cytochrome P450 2D6 gene. Primers and amplification conditions for the PCR reactions were provided by the manufacturer and protocols were performed following the test instructions. The main steps-fragmentation, labeling, hybridization, staining and scanning-were performed following the manufacturer's instructions. The AmpliChip CYP450 ${ }^{\circledR}$ array contains more than 15,000 oligonucleotide probes by which to analyze both sense and antisense strands of an amplified target DNA sample. It is able to distinguish 29 polymorphisms in the CYP2D6 gene, including gene deletion and duplication, and it allows the detection of 33 CYP2D6 alleles (i.e., *1 to *10AB, *11, *14A, *14B, *15, $* 17, * 19, * 20, * 25, * 26, * 29$ to $* 31, * 35, * 36, * 40, * 41$, $* 1 x N, * 2 x N, * 4 x N, * 10 x N, * 17 x N, * 35 x N$, and *41xN).

\section{Statistical analysis}

Kaplan-Meier estimates and the log-rank test were employed in univariate analysis of DFS. A Cox-regression

Table 2 Tamoxifen adverse effects

\begin{tabular}{lc}
\hline Adverse effects & $n(\%)$ \\
\hline Severe toxicity & $2(2.2)$ \\
Cerebrovascular accident & $3(3.3)$ \\
Pulmonary embolism & $2(2.2)$ \\
Deep vein thrombosis & $3(3.3)$ \\
Endometrial carcinoma & $1(1.0)$ \\
Toxicodermia & $11(12.0)$ \\
& \\
Other toxicities & $11(12.1)$ \\
Intrauterine polyp & $12(13.1)$ \\
Transaminitis & $3(3.3)$ \\
Amenorrhea & $3(3.3)$ \\
Ovaric cists & $2(2.2)$ \\
Steatosis & $2(2.2)$ \\
Ocular problems & $1(1.0)$ \\
Vaginitis & $34(37.2)$ \\
\end{tabular}

Hot flashes were not registered in the clinical records as an adverse effect model was used for DFS multivariate analysis. The results were considered to be statistically significant when bilateral $P$ values were less than 0.05 .

\section{Results}

Patient characteristics

The cohort was composed of 91 patients. Table 3 shows the clinical data of the patients included in the study. The median age of the cohort was 51 years (range, 2879 years). All tumours were estrogen-receptor positive. Most patients $(43 \%)$ presented with stage II disease. Half had positive axillary lymph nodes. Fifty patients $(55 \%)$ were treated with a modified radical mastectomy while breast-conserving surgery was performed in the remaining cases. The mean follow-up time was 108 months (range, 91-133 months).

\section{CYP2D6 genotype profiles}

The frequencies of individual CYP2D6 genotypes are shown in Table 4 . Null alleles $(* 3, * 4, * 5, * 6$, and 20), dysfunctional alleles $(9, * 10$, and 41$)$ and the remaining identified functional alleles $(* 1, * 2$, and $* 35)$ were designated as PM, IM, and EM alleles, respectively. Multiple copies of any functional allele were designated as UM.

\section{CYP2D6 genotype and clinical outcome}

No significant correlations were found between genotypes and clinical variables of prognostic relevance, such as

Table 3 Clinical characteristics of patients included in the study

\begin{tabular}{ll}
\hline $\begin{array}{l}\text { Median age } \\
\text { Years (range) }\end{array}$ & $5(28-79)$ \\
Menopausal status & \\
$\quad$ Premenopausal & $39(42.8 \%)$ \\
Postmenopausal & $36(39.6 \%)$ \\
$\quad$ Perimenopausal & $16(17.6 \%)$ \\
Disease status & \\
$\quad$ Stage I & $30(32.9 \%)$ \\
$\quad$ Stage II & $39(42.8 \%)$ \\
$\quad$ Stage III & $22(24.3 \%)$ \\
Operative procedure & \\
$\quad$ Mastectomy & $50(54.9 \%)$ \\
Breast-conserving & $41(45.1 \%)$ \\
Adjuvant therapy & \\
$\quad$ Monotherapy with tamoxifen & $33(39.8 \%)$ \\
Tamoxifen/chemotherapy & $58(60.2 \%)$ \\
\hline
\end{tabular}


Table 4 Genotypes for CYP2D6 and associated phenotypes

\begin{tabular}{lcl}
\hline CYP2D6 genotype & $n(\%)$ & CYP2D6 metabolizer status \\
\hline $4 / 4$ & $5(5.5)$ & PM/PM \\
$3 / 4$ & $1(1.1)$ & \\
$4 / 41$ & $2(2.2)$ & PM/IM \\
$4 / 9$ & $2(2.2)$ & \\
$9 / 10$ & $4(4.4)$ & IM/IM \\
$9 / 41$ & $1(1.1)$ & \\
$41 / 41$ & $1(1.1)$ & \\
$1 / 4$ & $9(9.9)$ & EM/PM \\
$1 / 5$ & $7(7.7)$ & \\
$1 / 6$ & $1(1.1)$ & \\
$2 / 4$ & $2(2.2)$ & \\
$2 / 5$ & $2(2.2)$ & \\
$2 / 20$ & $1(1.1)$ & \\
$35 / 4$ & $1(1.1)$ & \\
$1 / 10$ & $3(3.3)$ & EM/IM \\
$1 / 9$ & $3(3.3)$ & \\
$1 / 41$ & $7(7.7)$ & \\
$35 / 10$ & $1(1.1)$ & \\
$2 / 9$ & $2(2.2)$ & \\
$2 / 41$ & $3(3.3)$ & \\
$35 / 41$ & $1(1.1)$ & \\
$35 / 9$ & $1(1.1)$ & \\
$1 / 1$ & $11(12.1)$ & EM/EM \\
$1 / 2$ & $10(11.0)$ & \\
$1 / 35$ & $3(3.3)$ & \\
$2 / 2$ & $4(4.4)$ & \\
$2 / 35$ & $1(1.1)$ & \\
$1 x n / 2$ & $1(1 ., 1)$ & $\mathrm{UM} / \mathrm{EM}$ \\
$2 x n / 41$ & & \\
\hline & & \\
& & \\
& &
\end{tabular}

tumor size, nodal status, stage, and histologic grade. Eighty-three patients were evaluated for disease-free survival. Patients were grouped according to their CYP2D6 metabolizer status (Table 4) as follows: Group 1 (PM/PM and PM/IM); Group 2 (IM/IM, EM/PM, EM/IM) and Group 3 (EM/EM, UM/EM and UM/IM). No statistically significant differences were found in DFS between these groups $(P=0.413)$. Nevertheless, we observed a gradual improvement in DFS in function of the CYP2D6 genotype. Group 1 patients (poor metabolizers) had a mean DFS of 98 months; those in group 2 (intermediate metabolizers) had a mean DFS of 114 months, and group 3 patients (extensive metabolizers) had a mean DFS of 118 months.

When patients were grouped into group A $(* 4 / * 4, * 4 / * 41$, $* 1 / * 5$ and $* 2 / * 5$ ) and group B (the remaining genotypes), a significant difference in DFS was observed between groups $(P=0.016)$. The mean DFS was 95 months in group A and 119 months in group B (Fig. 1).
CYP2D6 genotype and toxicity

Adverse events related to tamoxifen therapy were evaluated in 91 patients (Table 2). Eleven patients (12\%) presented life-threatening toxicity, leading to discontinuation of tamoxifen. Thirty-four patients $(37.4 \%)$ presented mild toxicity, with transaminitis and uterine polyps being the most common. Forty-six patients $(50.5 \%)$ tolerated tamoxifen well. To perform the statistical analysis we considered the same genotype groups that give significant differences in DFS. This genotype classification was studied in relation to presentation of severe, mild, and no toxicity. We did not observe a significant relationship between genotype and toxicity $(P=0.2)$. Nevertheless, severe and mild toxicities were more frequent among poor metabolizer patients than among patients with a normal metabolizing pattern (18.8 and $43.8 \%$ vs. 10.7 and $36 \%$, respectively; Table 5).

\section{Discussion}

The four distinct CYP2D6 phenotypes are commonly referred to as ultrarapid, extensive, intermediate and poor metabolizers and they make up about $3-5,70-80,10-15$ and $7-10 \%$ of the Caucasian population $[4,7,10]$. In this study, the frequencies of the CYP2D6 genotypes, inferred from the different allele frequencies, were similar: $3 \%$ of UMs, $78 \%$ of EMs, $12 \%$ of IMs and $7 \%$ of PMs.

Previous studies have evaluated the association between CYP2D6 genotype and clinical outcomes in women treated with adjuvant tamoxifen [11-15]. Two studies [14, 15] retrospectively enrolled relatively homogeneous populations

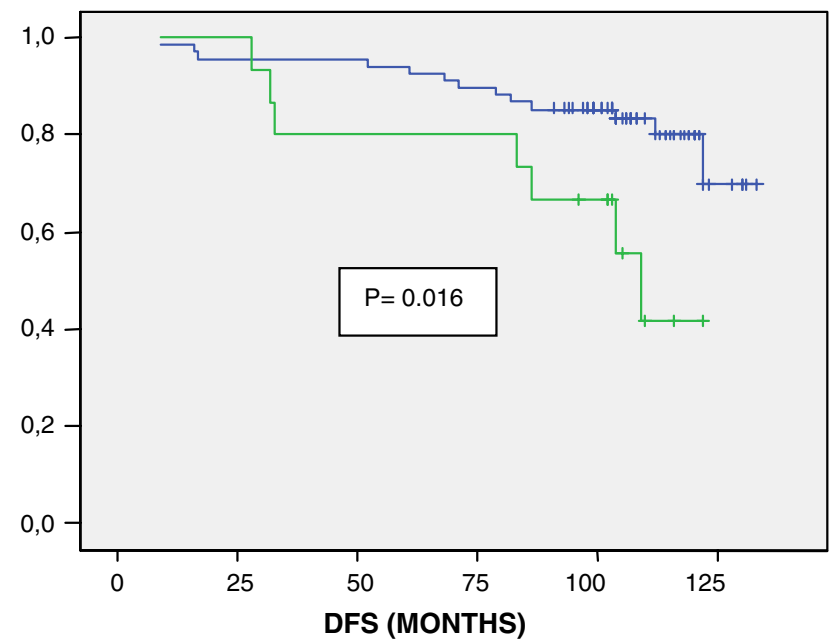

Fig. 1 Kaplan-Meier probabilities of disease-free survival of patients as a function of their CYP2D6 genotype. Lower line: patients with $* 4$ / $* 4, * 4 / * 41, * 1 / * 5$ and $* 2 / * 5$ CYP2D6 genotype. Upper line: patients with the remaining genotypes $(P=0.016)$ 
Table 5 Relationships between CYP2D6 genotype and toxicitiy

\begin{tabular}{lrcl}
\hline & No toxicity & Mild toxicity & Severe toxicity \\
\hline$* 4 / * 4, * 4 / * 41, * 1 / * 5$ and $* 2 / * 5$ & $6(37.5 \%)$ & $7(43.8 \%)$ & $3(18.8 \%)$ \\
Remaining genotypes & $40(53.3 \%)$ & $27(36 \%)$ & $8(10.7 \%)$ \\
\hline
\end{tabular}

of patients who were estrogen-receptor positive. The studies differed in that Goetz et al. [14] only genotyped for the CYP2D6*4 nonfunctional variant, whereas Schroth et al. $[15]$ also tested for the $* 5$ nonfunctional variant, and for the $* 10$ and $* 41$ reduced function variants. Evidence from these two studies suggests that women treated with tamoxifen who are functional poor or intermediate CYP2D6 metabolizers have a significantly shorter time to recurrence and recurrence-free survival (but not overall survival) as compared to EMs. Few variant alleles were typed in these studies, and samples that were negative for variant alleles were assumed to be wild-type EMs; more extensive genotyping and better categorization might strengthen these results.

Wegman et al. [12] reported that a small subset of patients with estrogen receptor (ER)-positive breast cancer treated with tamoxifen who carried the CYP2D6*4 variant allele did significantly better than patients with the same variants not treated with tamoxifen. This study that reported conflicting evidence has two serious limitations: the numbers are very small and only one null allele of the CYP2D6 gene has been investigated.

As we used the AmpliChip CYP450 GeneChip ${ }^{\circledR}$, Roche, most of the different genotypes were covered and an accurate determination of the CYP2D6 status was achieved. Although the present work has some limitations (the retrospective bias selection of patients, the sample size, the possible use of co-administered inhibitors and the infrequency of the CYP2D6 variants in the European population) our results clearly show a significant association between an absent or reduced enzyme function-homozygous or compound heterozygous for CYP2D6 alleles $* 4$, $* 5$, and $* 41$ - and worse disease-free survival. Benefits in event-free survival were similar to those found by Schroth and Goetz $[14,15]$. The fact that CYP2D6 poorer metabolizers have a worse clinical outcome than better metabolizers could be related to a reduced tamoxifen metabolism and lower endoxifen levels in the former; if we assume that a certain level of endoxifen is sufficient and necessary for tamoxifen efficacy, this level would not be reached by neither any CYP2D6 poor metabolizer nor by some of the patients with an intermediate metabolizer genotype.

Regarding the relationships between the CYP2D6 genotype and the occurrence of side effects caused by tamoxifen, we observed that severe, and mild toxicities tended to be more frequent among poor metabolizer patients than in patients with a normal metabolizer pattern. This finding could suggest that an increased concentration of a tamoxifen metabolite may be related with the development of mild and severe toxicities. However, as no information was available about co-administration of antidepressants in our group of patients, no definitive conclusion could be reached about the correlation between genotype and toxicity.

The FDA is considering updating tamoxifen labelling with information or recommendations regarding CYP2D6 genotyping and its impact on the efficacy of the drug. On October 18, 2006, an FDA Advisory Committee meeting was held to answer specific questions regarding evidence and recommendations for the label update. I was recommended that information should be included about CYP2D6 genotypes and the potential effect on patient outcomes, as well as information on CYP2D6 genotyping tests. The members did not reach a consensus as to whether testing should be recommended or considered as an option. Since this Advisory Committee meeting, AstraZeneca, the brand name $\left(\right.$ Nolvadex $\left.^{\circledR}\right)$ manufacturer, has ceased to produce tamoxifen. When this paper was submitted for publication, the FDA had still not issued a statement regarding labelling of generic versions of tamoxifen to include CYP2D6 genotyping information.

Additional studies with inclusion of concomitant medications are needed to verify and extend initial results, and to accurately identify genotypes associated with poorer outcomes in patients treated with tamoxifen. In the meanwhile, aromatase inhibitors in post-menopausal women and possibly ovarian suppression in premenopausal patients can be considered as alternatives to tamoxifen use in patients with a poor metabolizer genotype.

\section{References}

1. Osborne CK (1998) Tamoxifen in the treatment of breast cancer. N Engl J Med 339:1609-1618. doi:10.1056/NEJM19981126339 2207

2. Early Breast Cancer Trialists' Collaborative Group (EBCTCG) (2005) Effects of chemotherapy and hormonal therapy for early breast cancer on recurrence and 15-year survival: an overview of the randomized trials. Lancet 365:1687-1717. doi:10.1016/ S0140-6736(05)66544-0

3. Stearns V, Johnson MD, Rae JM et al (2003) Active tamoxifen metabolite plasma concentrations after coadministration of tamoxifen and the selective serotonin reuptake inhibitor paroxetine. J Natl Cancer Inst 95:1758-1764

4. Sachse C, Brockmöller J, Bauer S et al (1997) Cytochrome P450 2D6 variants in a Caucasian population: allele frequencies and phenotypic consequences. Am J Hum Genet 60:284-295 
5. Kubota T, Yamaura Y, Ohkawa N et al (2000) Frequencies of CYP2D6 mutant alleles in a normal Japanese population and metabolic activity of dextromethorphan O-demethylation in different CYP2D6 genotypes. Br J Clin Pharmacol 50:31-34. doi: 10.1046/j.1365-2125.2000.00209.x

6. Griese EU, Asante-Poku S, Ofori-Adjei D et al (1999) Analysis of the CYP2D6 gene mutations and their consequences for enzyme function in a West African population. Pharmacogenetics 9:715-723. doi:10.1097/00008571-199912000-00006

7. Menoyo A, del Rio E, Baiget M (2006) Characterization of variant alleles of cytochrome CYP2D6 in a Spanish population. Cell Biochem Funct 24:381-385. doi:10.1002/cbf.1258

8. Home Page of the Human Cytochrome P450 (CYP) Allele Nomenclature Committee (www.cypalleles.ki.se)

9. Miller SA, Dykes DD, Polesky H (1989) A simple salting out procedure for extracting DNA from human nucleated cells. Nucleic Acids Res 16:1215. doi:10.1093/nar/16.3.1215

10. Raimundo S, Toscano C, Klein K et al (2004) A novel intronic mutation, $2988 \mathrm{G}>$ A, with high predictivity for impaired function of cytochrome P450 2D6 in white subjects. Clin Pharmacol Ther 76:128-138. doi:10.1016/j.clpt.2004.04.009
11. Nowell SA, Ahn J, Rae JM et al (2005) Association of genetic variation in tamoxifen-metabolizing enzymes with overall survival and recurrence of disease in breast cancer patients. Breast Cancer Res Treat 91:249-258. doi:10.1007/s10549-004-7751-x

12. Wegman P, Vainikka L, Stal O et al (2005) Genotype of metabolic enzymes and the benefit of tamoxifen in postmenopausal breast cancer patients. Breast Cancer Res 7:R284-R290. doi: $10.1186 /$ bcr993

13. Goetz MP, Rae JM, Suman VJ et al (2005) Pharmacogenetics of tamoxifen biotransformation is associated with clinical outcomes of efficacy and hot flashes. J Clin Oncol 23:9312-9318. doi: 10.1200/JCO.2005.03.3266

14. Goetz MP, Knox SK, Suman VJ et al (2007) The impact of cytochrome P450 2D6 metabolism in women receiving adjuvant tamoxifen. Breast Cancer Res Treat 101:113-121. doi:10.1007/ s10549-006-9428-0

15. Schroth W, Antoniadou L, Fritz P et al (2007) Breast cancer treatment outcome with adjuvant tamoxifen relative to patient CYP2D6 and CYP2C19 genotypes. J Clin Oncol 25:5187-5193. doi:10.1200/JCO.2007.12.2705 